\title{
Determinación del contenido de compuestos de cromo en el cemento portland por espectrofotometría de absorción atómica Segunda parte
}

\author{
DEMETRIO GASPAR-TEBAR, FERNANDO TRIVIÑO-VAZQUEZ y VICENTE ALVAREZ-PUENTE
}

\section{INTRODUCCION}

En un trabajo anterior (1) se ha puesto de manifiesto la importancia que tiene conocer el contenido de compuestos de cromo (III) y de cromo (VI) en el cemento portland, habiendo determinado dicho contenido en diversas muestras de distintos cementos, fabricados por la industria española, por espectrofotocolorimetría del complejo coloreado formado en la reacción que tiene lugar entre el cromo (VI) y la s-difenilcarbacida, de tal modo que se puedan utilizar como patrones para estudiar y poner a punto un procedimiento rápido y preciso que utiliza la espectrofotometría de absorción atómica, objeto del presente artículo.

La espectrofotometría de absorción atómica se viene aplicando con éxito en el campo de la industria del cemento para determinar la composición química del cemento portland y de sus materias primas (2) (3) (4) (5) (6) (7) (8), etc., especialmente de los iones Ca (II), $\mathrm{Mg}$ (II), Al (III), Fe (III), Si (IV), Mn (II), Sr (II), Ti (IV), Na (I) y K (I), con una precisión igual o superior a la obtenida cuando se usan las técnicas químicas clásicas, excepto para el $\mathrm{Ca}$ (II) y a veces para el $\mathrm{Si}$ (IV).

La longitud de onda más sensible del pico de absorción del cromo es $357,9 \mathrm{~nm}$ (7) (9) y $359 \mathrm{~nm}(10)$; el límite de deteción es $0,05 \mu \mathrm{g} / \mathrm{ml}$ (9), 0,005 $\mu \mathrm{g} / \mathrm{ml}$ (7), 0,01 ppm (10) y la sensibilidad $0,15 \mu \mathrm{g} / \mathrm{ml} / 1 \%$.

La disolución de la muestra de cemento se hace por ataque con ácidos minerales o por fusión alcalina. La fusión con $\mathrm{LiBO}_{2}$, utilizada en absorción atómica (8) (11) (12) (13) (14) (15) (16), es simple, segura y rápida, presentando, además, las ventajas mencionadas en (1).

Degré (16) en su trabajo sobre automatización del análisis elemental cuantitativo, por espectrofotometría de absorción atómica, aplicado a diferentes materias primas y productos de la industria del cemento, utiliza como fundente $\mathrm{LiBO}_{2}$ y para eliminar las interferencias La (III); en las tablas de valores de las sustancias clinkerizadas, escoria y bauxita, que figuran en dicho trabajo señala el contenido de cromo. 


\section{PARTE EXPERIMENTAL}

La falta de información sobre la determinación cuantitativa de los compuestos de cromo en el cemento portland por medio de la espectrofotometría de absorción atómica, técnica instrumental que se usa en la industria del cemento, nos ha llevado a estudiar y poner a punto un procedimiento que permita dicha determinación por el método de lectura directa; los resultados obtenidos confirman los hallados por otra técnica de referencia -además de la espectrofotocolorimétrica- método de las adiciones patrón, recomendado para el análisis de elementos traza en presencia de elementos mayoritarios, que presenta la ventaja de tener la misma matriz para las distintas muestras y, por consiguiente, los efectos interelementales se compensan automáticamente.

\subsection{Equipo y condiciones de trabajo}

Se ha utilizado un espectrómetro de absorción atómica Perkin-Elmer modelo 305, con lector digital Hitachi-Perkin-Elmer, modelo UDR-2.

Condiciones de trabajo:

- Lámpara de cromo, de cátodo hueco, de alta intensidad (25 mA).

- Longitud de onda: $357,9 \mathrm{~nm}$.

- Rendija de radiación: 0,2 nm, posición 3 .

- Constante de tiempo: posición 1.

- Ganancia: posición 1.

- Expansión de escala: 2 vueltas.

- Altura de mechero: 10.

- Llama: aire- $\mathrm{C}_{2} \mathrm{H}_{2}$.

— Flujo de aire: $11,5 \mathrm{l} / \mathrm{min}$.

- Flujo de $\mathrm{C}_{2} \mathrm{H}_{2}: 6,5 \mathrm{l} / \mathrm{min}$.

\subsection{Disoluciones y reactivos empleados}

- Disolución patrón de cromo (VI), con una concentración de 0,1 g por litro de disolución.

La disolución patrón de cromo (VI) se ha preparado disolviendo, en agua desionizada, $0,282_{9} \mathrm{~g}$ de $\mathrm{K}_{2} \mathrm{Cr}_{2} \mathrm{O}_{7}$ Merck, p.a., previamente secado a $140^{\circ}-150^{\circ} \mathrm{C}$, que se lleva a 1.000 mililitros.

- Disolución de Lantano (III).

Se disuelven 58,600 $\mathrm{g}$ de $\mathrm{La}_{2} \mathrm{O}_{3}$ de calidad "Atomic Absorption", en $500 \mathrm{ml}$ de $\mathrm{HCl}$ concentrado y, con agua desionizada, se lleva posteriormente a un volumen de $1.000 \mathrm{ml}$. Se ha preparado otra disolución de La (III) en medio $\mathrm{HNO}_{3}$, que no se ha utilizado, a pesar de eliminar la interferencia del hierro (III), por producir un descenso considerable en la absorción. 
- Disolución de hierro (III), con una concentración de 537 mg por litro de disolución.

La disolución de $\mathrm{Fe}$ (III) se ha preparado disolviendo $0,767_{8}$ g de $\mathrm{Fe}_{2} \mathrm{O}_{3}$ Merck, p.a., en $15 \mathrm{ml}$ de agua desionizada y $30 \mathrm{ml}$ de $\mathrm{HCl}$ concentrado que se han llevado posteriormente a $1.000 \mathrm{ml}$.

- Metaborato de litio.

Se ha utilizado $\mathrm{LiBO}_{2}$, Phaxe, de calidad "Atomic Absorption". Se ha comprobado que no produce interferencias.

\subsection{Muestras de cemento estudiadas}

Se han estudiado, por una parte, las diez muestras de cemento reseñadas en (1) y además tres muestras de clínkeres distintas (núms. 12, 13 y 14) y una nueva de cemento portland, (núm. 11) fabricados por la industria española, que responden a la composición química que se incluye en la tabla 1, determinada de acuerdo con las técnicas de trabajo descritas en el apartado 2.7 del PCCH-64 (19). El contenido de elementos alcalinos, $\mathrm{Na}$ (I) y K (I), se ha efectuado por espectrofotometría de llama.

TAB LA 1

Composición química de las muestras estudiadas

\begin{tabular}{|c|c|c|c|c|c|}
\hline \multirow{2}{*}{ Determinación } & & \multicolumn{4}{|c|}{ Muestra n.o } \\
\hline & & 11 & 12 & 13 & 14 \\
\hline Pérdlida por calcinación ... & PF & 1,2 & 1,0 & 1,2 & 0,9 \\
\hline Residuo insoluble $\ldots \ldots \ldots$ & RI & $0, \overline{2}$ & 0,3 & 0,0 & 0,3 \\
\hline Dióxido de silicio $\ldots \ldots \ldots$ & $\mathrm{SiO}_{2}$ & 21,3 & 19,1 & 18,7 & 19,3 \\
\hline Oxido de Aluminio $\ldots \ldots$ & $\mathrm{Al}_{2} \mathrm{O}_{3}$ & 2,1 & 5,8 & 5,6 & 5,8 \\
\hline $\begin{array}{lllll}\text { Oxido férrico } & \ldots & \ldots & \ldots & \ldots\end{array}$ & $\mathrm{Fe}_{2} \mathrm{O}_{3}$ & 4,5 & 3,5 & 3,3 & 3,3 \\
\hline Oxido cálcico $\ldots \begin{array}{llll} & \ldots & \ldots & \ldots\end{array}$ & $\mathrm{CaO}$ & 67,5 & 62,1 & 63,2 & 61,8 \\
\hline Oxido magnésico $\ldots \ldots \ldots$ & $\mathrm{MgO}$ & 0,5 & 4,9 & 4,7 & 4,9 \\
\hline Trióxido de azufre $\ldots \ldots$ & $\mathrm{SO}_{3}$ & 2,5 & 1,9 & 2,1 & 2,4 \\
\hline $\begin{array}{lllll}\text { Oxido sódico } & \ldots & \ldots & \ldots & \ldots\end{array}$ & $\mathrm{Na}_{2} \mathrm{O}$ & s.d. & 0,5 & 0,5 & 0,5 \\
\hline $\begin{array}{lllll}\text { Oxido potásico } & \ldots & \ldots & \ldots & \ldots\end{array}$ & $\mathrm{K}_{2} \mathrm{O}$ & s.d. & 0,7 & 0,7 & 0,6 \\
\hline Suma $\ldots \ldots$ & $\ldots \ldots \ldots$ & 99,8 & 99,8 & 100,0 & 99,8 \\
\hline
\end{tabular}

El contenido de los distintos iones determinados, referido a la muestra seca a $105^{\circ}-110^{\circ} \mathrm{C}$, se ha expresado, en \% en peso, como óxidos.

\subsection{Preparación de la muestra}

Las operaciones fundamentales para preparar la muestra las hemos dividido en dos grupos: disolución y eliminación de interferencias. 


\section{Disolución}

La disolución de la muestra de cemento se ha realizado por fusión con $\mathrm{LiBO}_{2}$ en crisol de grafito, siguiendo la técnica descrita en (1).

\section{Eliminación de interferencias}

Barnes (2) y Giammarise (3) señalan que para determinar el cromo en aleaciones, trabajando con llama aire- $\mathrm{C}_{2} \mathrm{H}_{2}$, interfiere la presencia de níquel y de hierro. La concentración de compuestos de níquel en los cementos portland es prácticamente despreciable, por lo que no se ha tenido en cuenta; por el contrario la concentración de hierro, cuyos límites normales se encuentran comprendidos entre 0,5 y 7,6 \%, como $\mathrm{Fe}_{2} \mathrm{O}_{3}$, que corresponde a 0,38 y $5,37 \%$ como hierro, influye en la determinación del cromo que, como hemos comprobado, se encuentra en cantidades que oscilan, normalmente, entre 10 y 100 ppm. La eliminación de la interferencia del hierro la realizan, dichos investigadores, por adición de $2 \mathrm{~g}$ de $\mathrm{NH}_{4} \mathrm{Cl}$ por cada $100 \mathrm{ml}$ de disolución.

La eliminación de interferencias químicas en el análisis de cementos por espectrofotometría de absorción atómica se hace normalmente por adición de La (III) a la disolución de la muestra (6) (7) (8) (12) (14) (15) (16) (17) (18); de aquí que para eliminar la interferencia del $\mathrm{Fe}$ (III) se haya estudiado la influencia del $\mathrm{NH}_{4} \mathrm{Cl}$ y del $\mathrm{La}$ (III) en una sèrie de disoluciones de $\mathrm{Cr}$ (VI) de concentración conocida.

\subsection{Técnica de trabajo}

Se toman $0,5 \mathrm{~g}$ de la muestra de cemento seca a $105^{\circ}-110^{\circ} \mathrm{C}$, pesados hasta $\pm 0,0001 \mathrm{~g}$, que se mezclan íntimamente en un mortero de ágata con $0,5 \mathrm{~g}$ de $\mathrm{LiBO}_{2}$. La mezcla se introduce en un crisol de grafito, se cubre con $0,25 \mathrm{~g}$ de $\mathrm{LiBO}_{2}$, se tapa con una placá de grafito y a continuación se funde, a $1.000^{\circ} \mathrm{C}$ durante $3-5$ minutos, en un horno; se destapa el crisol y se le imprime un movimiento de rotación hasta formación de una perla; se tapa nuevamente el crisol y se continúa la calefacción durante otros 3-5 minutos.

El contenido del crisol se vacía sobre un vaso de precipitados de forma alta, de $250 \mathrm{ml}$, que contiene $10 \mathrm{ml}$ de la disolución de La (III), $20 \mathrm{ml}$ de agua desionizada y $10 \mathrm{ml}$ de $\mathrm{HCl}$ concentrado. Se agita el fundido del crisol, sin calentar, hasta conseguir la disolución; se filtra y se lleva a un volumen de $50 \mathrm{ml}$ o de $125 \mathrm{ml}$ con agua desionizada, según se utilice el método de lectura directa o el de las adiciones patrón.

\section{Método de las adiciones patrón}

A partir de los $125 \mathrm{ml}$ de la disolución, obtenida como se ha mencionado anteriormente, se toman 4 partes alícuotas de $25 \mathrm{ml}$ a los que se añaden $0,0-5,0-10,0$ y $15,0 \mathrm{ml}$, respectivamente, de la disolución patrón de cromo (VI) que tiene $1,0 \mathrm{mg} / \mathrm{l}$ y se lleva a un volumen de $50 \mathrm{ml}$. A continuación se obtiene el \% de absorción, por espectrofotometría de absorción atómica, de cada disolución y el de otra disolución que contiene todos los reactivos, excepto el cromo, para determinar el fondo espectral de absorción.

El contenido de cromo en la muestra objeto /de estudio, que corresponde a la parte alícuota sin adición de la disolución-patrón de $\mathrm{Cr}$ (VI), se determina gráficamente por extrapolación (figura 4). 
La disolución obtenida como se ha dicho en el apartado anterior, $50 \mathrm{ml}$, está preparada para determinar por espectrofotometría el contenido de cromo; para ello hace falta tener la correspondiente curva de calibrado.

\section{RESULTADOS}

\subsection{Eliminación de la interferencia del Fe (III)}

Para estudiar la eliminación de la interferencia del Fe (III) se han preparado, en una primera etapa, dos series de disoluciones de $\mathrm{Cr}$ (VI) de concentración conocida: una se ha utilizado como testígo y otra, a la que se le ha adicionado $537 \mathrm{mg} / l$ de $\mathrm{Fe}$ (III)*, cantidad máxima frecuente en los cementos portland corrientes, para conocer la influencia que ejerce el Fe (III) en la absorción (\%) de las distintas disoluciones de cromo.

Los resultados obtenidos se han representado en la figura 1, gráficas $\mathrm{Cr}$ (VI) y $\mathrm{Cr}$ (VI) + + Fe (III). La concentración de cromo de las distintas disoluciones ha sido 0,1, 0,3, 0,6 y $1,0 \mathrm{mg} / l$; cantidades máxima y mínima que se han tomado teniendo en cuenta el contenido de cromo en los cementos españoles estudiados en la parte (1), cantidades que están de acuerdo con los estudios de Valle (20) y con la información proporcionada por Keil (21).

Como puede apreciarse, la gráfica correspondiente a las disoluciones de cromo en el intervalo de concentraciones estudiado sigue la ley de Bouguer-Lambert-Beer. Sin embargo, la gráfica correspondiente a las disoluciones de $\mathrm{Cr}$ (VI) $+\mathrm{Fe}$ (III) presenta un punto de inflexión para la disolución que tiene una concentración de cromo de $0,6 \mathrm{mg} / l$, produciéndose a partir de este punto una disminución de la absorción (\%); la zona de la gráfica con concentraciones de cromo $\leqslant$ de $0,6 \mathrm{mg} / l$ presenta valores de absorción (\%) del mismo orden que para el caso de la disolución de cromo (VI).

Posteriormente se han considerado otras tres series de disoluciones, cada una con la misma concentración de $\mathrm{Cr}$ (VI), para estudiar la posibilidad de eliminar la interferencia del $\mathrm{Fe}$ (III), bien por adición de $\mathrm{NH}_{4} \mathrm{Cl}$ o de La (III).

La adición de $\mathrm{NH}_{4} \mathrm{Cl}, 20 \mathrm{~g}$ por litro de disolución (2) (3), elimina la interferencia producida por el $\mathrm{Fe}$ (III); la recta que se obtiene cumple la ley de Bouguer-Lambert-Beer, gráfica $\mathrm{Cr}(\mathrm{VI})+\mathrm{Fe}$ (III) $+\mathrm{NH}_{4} \mathrm{Cl}$ de la figura 1. Los valores de la absorción (\%) son análogos a los de la serie de $\mathrm{Cr}$ (VI).

La adición de La (III), 1 \%, cantidad normal empleada para eliminar interferencias químicas en el análisis de cemento por espectrofotometría de absorción atómica, a partir de la disolución de $\mathrm{La}_{2} \mathrm{O}_{3}(58,6 \mathrm{~g} / \mathrm{l})$, también elimina la interferencia química producida por el mencionado Fe (III) y causa, además, un incremento considerable de la absorción (\%) como puede apreciarse en la gráfica $\mathrm{Cr}$ (VI) + Fe (III) + La (III) de la figura 1. La gráfica cumple la ley de Bouguer-Lambert-Beer.

* Corresponde a $7,6 \%$ de $\mathrm{Fe}_{2} \mathrm{O}_{3}$ y a $5,37 \%$ de $\mathrm{Fe}$. 
La absorción (\%) de las disoluciones de la serie de Cr (VI), a las que se les ha añadido la misma cantidad de La (III) que en el caso anterior, es del mismo orden como puede apreciarse en la figura 1, gráfica $\mathrm{Cr}$ (VI) + La (III).

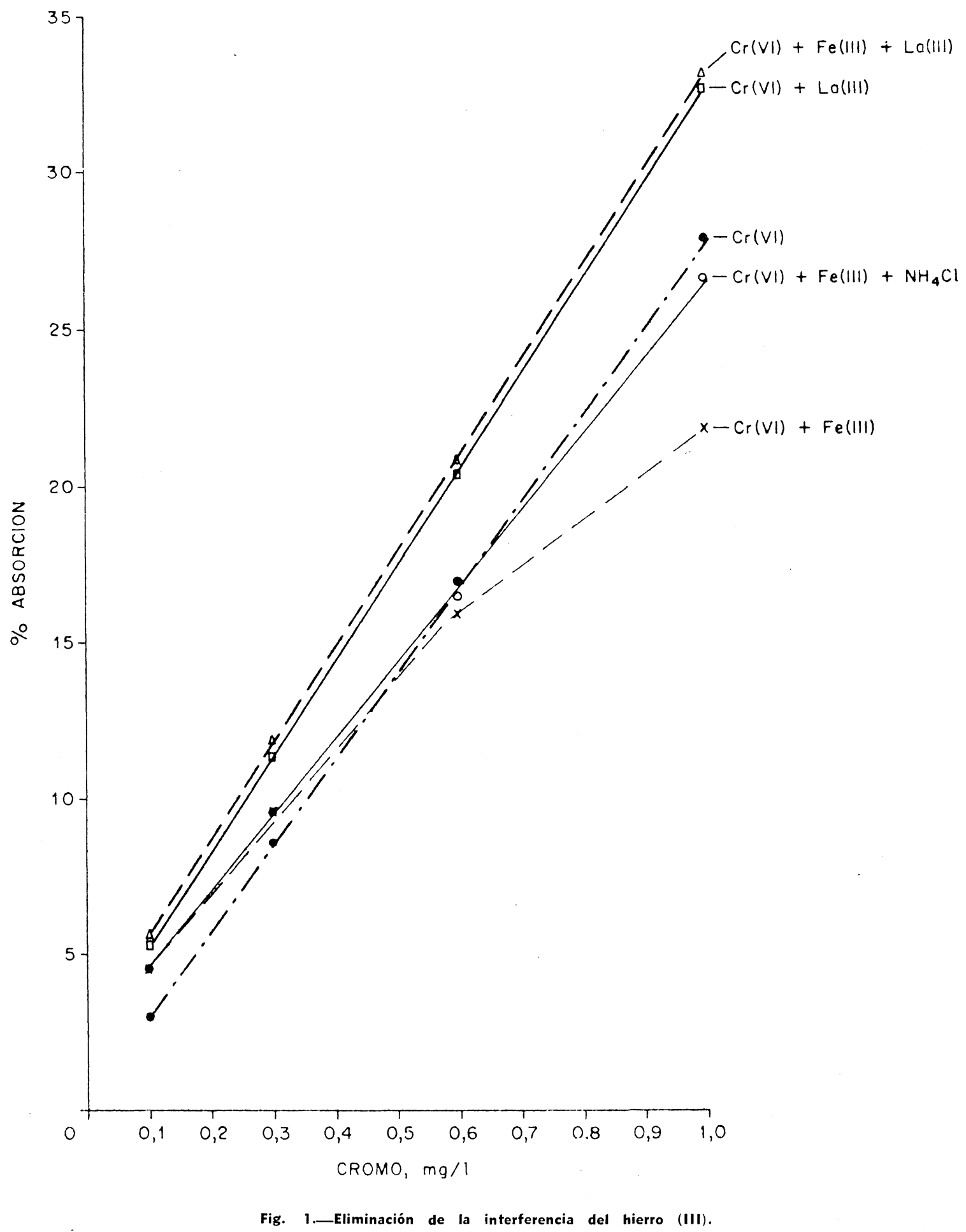

A la vista de los resultados obtenidos, se ha estudiado la influencia de la adición de La (III) para eliminar la interferencia química del $\mathrm{Fe}$ (III) en la determinación cuantitativa de compuestos de cromo en el cemento portland. 


\subsection{Influencia de la cantidad de La (III)}

Con objeto de determinar la influencia de la cantidad de La (III) se han preparado tres series de disoluciones de $\mathrm{Cr}$ (VI) que tienen una concentración de 0,1, 0,5 y $1,0 \mathrm{mg} / \mathrm{l}$, a las que se les ha adicionado $5,86,3,91,(5,86 / 1,5)$ y $1,95(5,86 / 3) \mathrm{g} / l$ de $\mathrm{La}_{2} \mathrm{O}_{3}$, respectivamente, que corresponden a 4.997, 3.334 y $1.663 \mathrm{~g} / l$ de La (III).

La adición máxima de La (III) corresponde, como se ha mencionado, a la que normalmente se utiliza para eliminar las interferencias químicas en el análisis del cemento portlan por absorción atómica (7) (23), debido a la formación de compuestos refractarios a base de aluminatos, silicatos y silicoaluminatos de calcio.

Los resultados obtenidos de las disoluciones de $\mathrm{Cr}$ (VI) y de $\mathrm{Cr}$ (VI) + La (III) se encuentran representados en las figuras 2 y 3 ; en ellas puede apreciarse que la adición creciente de La (III), para una misma cantidad de $\mathrm{Cr}$ (VI), exalta el valor de la absorción (\%) y que se obtiene un haz de rectas prácticamente paralelas para las tres adiciones de La (III) estudiadas (figura 3 ).

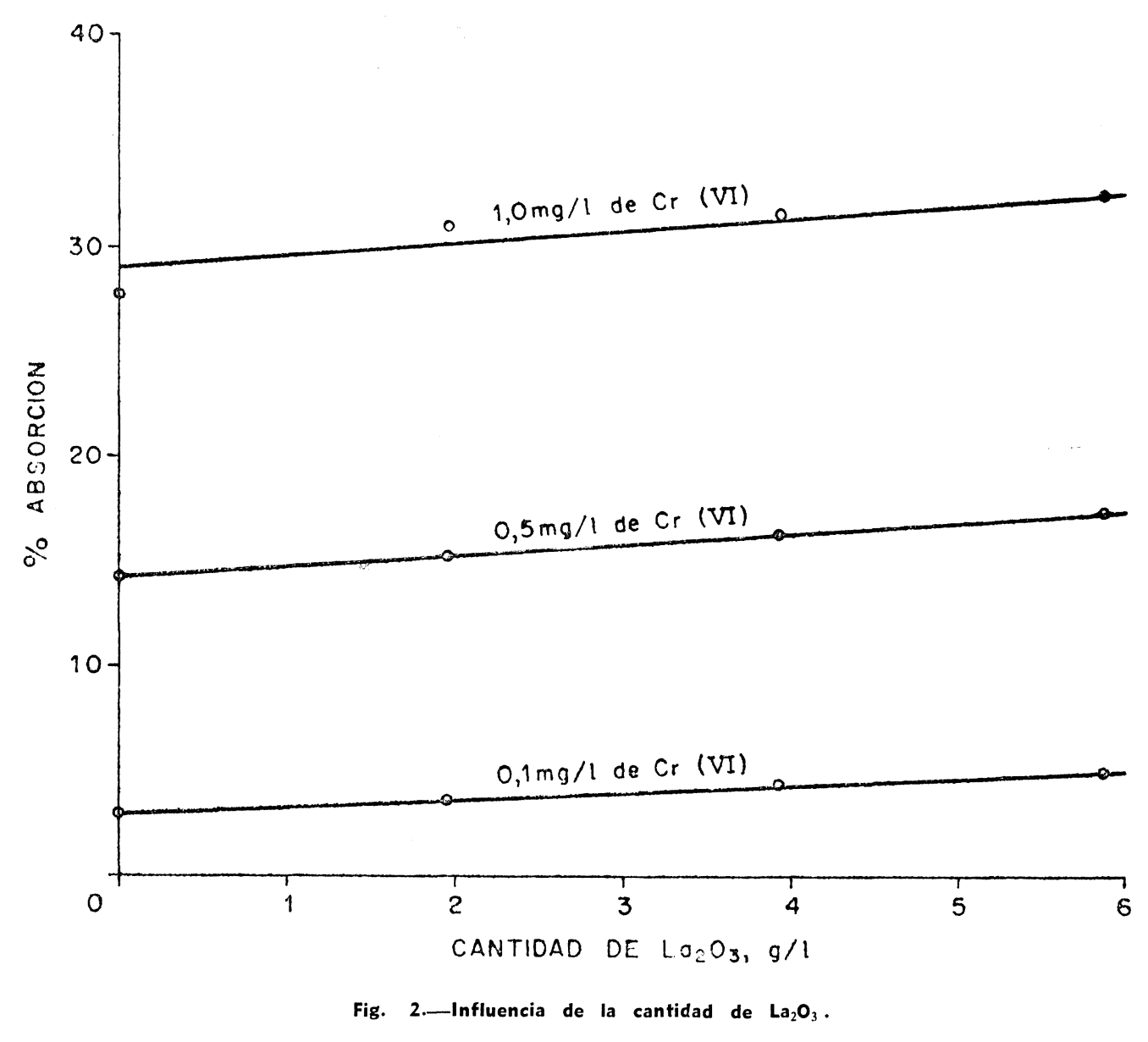

\subsection{Determinación del contenido de compuestos de cromo en diversas muestras de cemento}

El contenido total de compuestos de cromo, $\mathrm{Cr}$ (III) y Cr (VI), se ha determinado por el método de las adiciones (dadas las ventajas que presenta para determinar elementos tra- 
za y utilizar siempre la misma matriz) en las 10 muestras de cemento portland fabricadas por la industria española, que se reseñaron en (1) y por el método de lectura directa en 6 de dichas muestras.

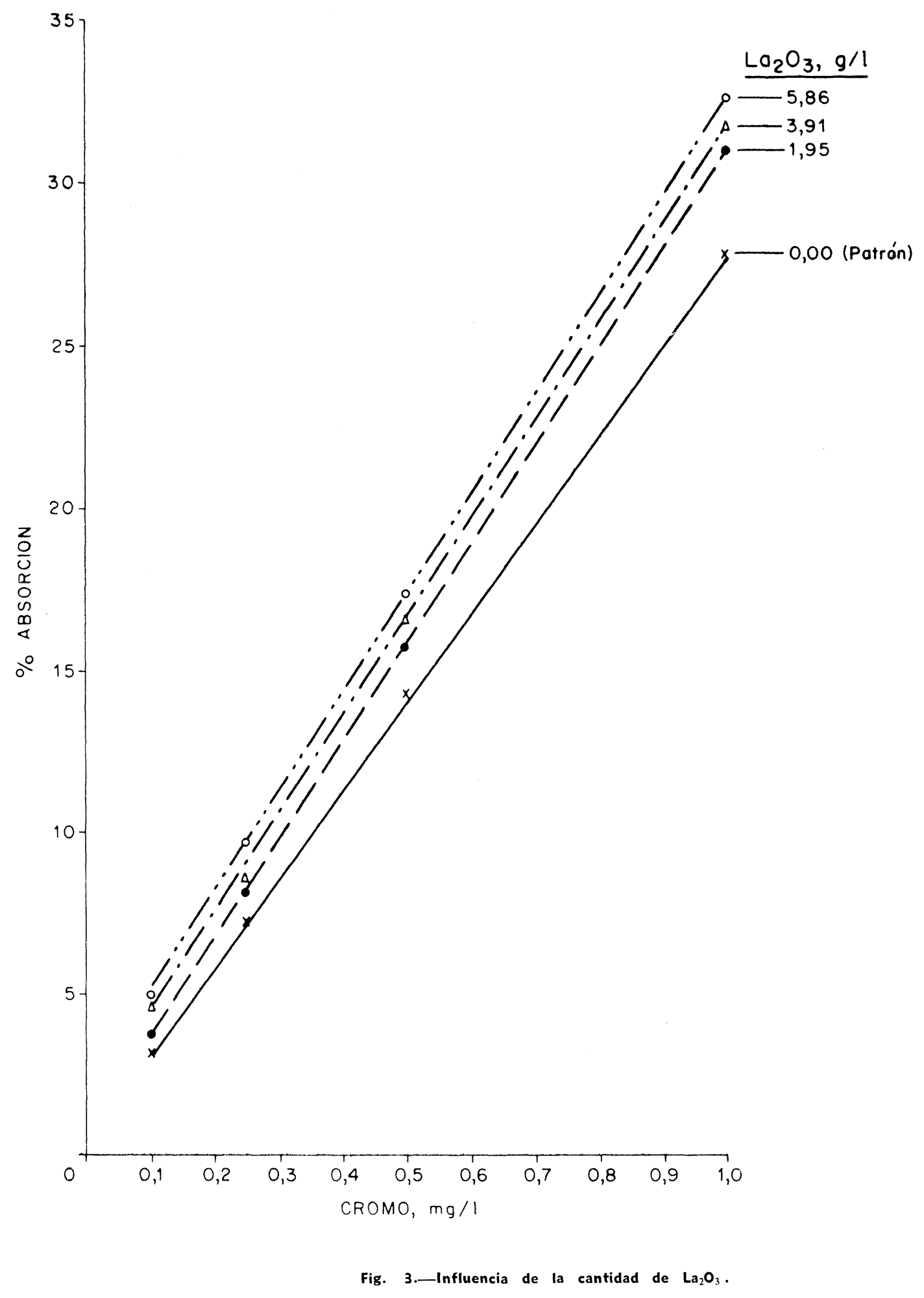

a) Determinación por el método de las adiciones patrón

Los resultados obtenidos, expresados en ppm y referidos a la muestra seca a $105^{\circ} \mathrm{C}-110^{\circ} \mathrm{C}$, son los que figuran en la tabla 2. 
TAB A 2

Contenido de cromo en 10 muestras de cemento

\begin{tabular}{|c|c|c|c|}
\hline Muestra $n .^{\circ}$ & Cromo ppm & Muestra $n .^{\circ}$ & Cromo ppm \\
\hline 1 & 96,5 & 6 & 70,3 \\
\hline 2 & 53,0 & 7 & 75,0 \\
\hline 3 & 35,5 & 8 & 48,0 \\
\hline 4 & 55,5 & 9 & 30,5 \\
\hline 5 & 74,0 & 10 & 55,0 \\
\hline
\end{tabular}

En la figura 4 se incluye, a título de ejemplo, el procedimiento gráfico seguido para una muestra n. ${ }^{\circ}$ 7. En ordenadas se han representado los $\%$ de absorción correspondientes a la muestra (x), sin adición de la disolución patrón de cromo (VI), a las muestras con una adición de $0,1,0,2$ y $0,3 \mathrm{mg}$ de cromo por litro de disolución $(\mathrm{x}+0,1 ; \mathrm{x}+0,2 \mathrm{y} \mathrm{x}+0,3$, respectivamente) y el de la disolución en blanco que contiene los reactivos utilizados, excepto el cromo de la disolución patrón, para obtener el fondo espectral de absorción; las concentraciones figuran en abscisas.

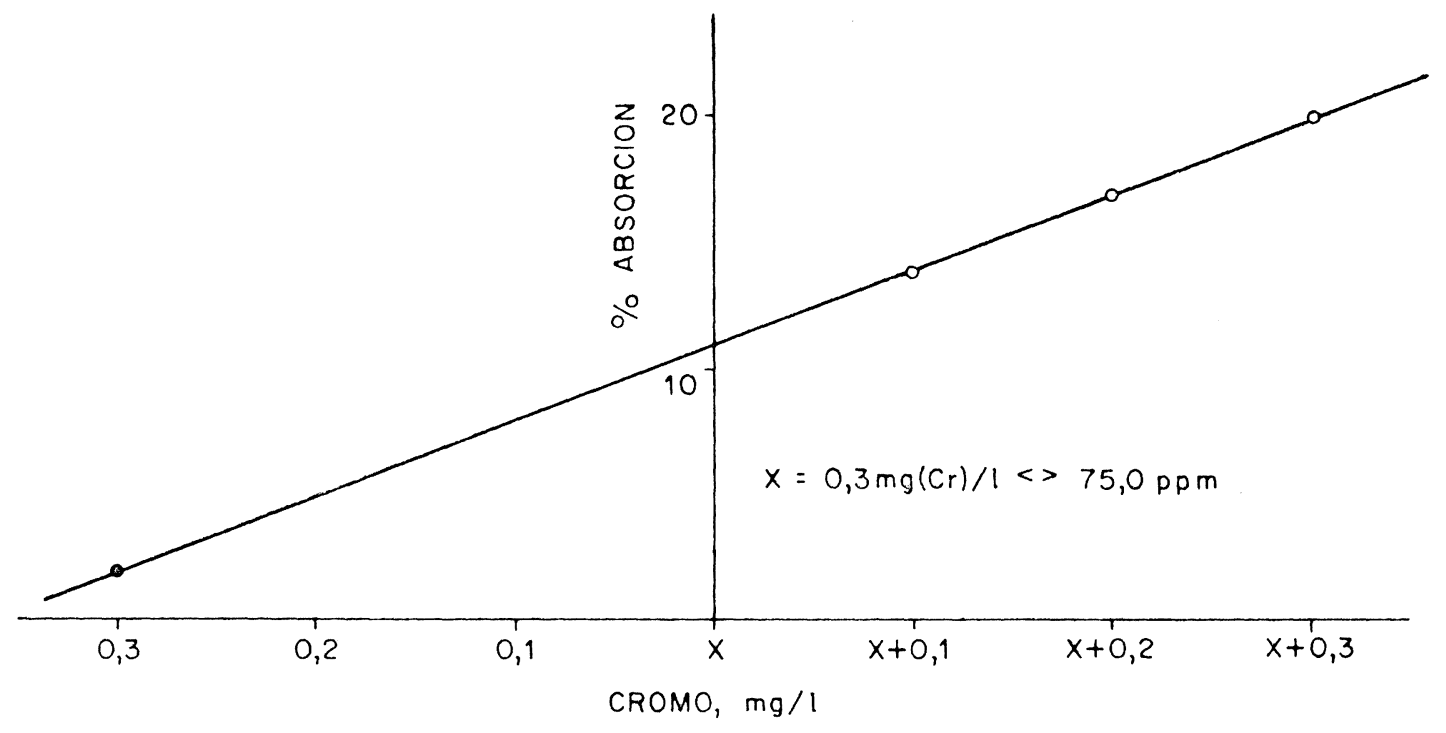

Fig. 4.-Método de las adiciones patrón.

El punto de corte de la recta que une los \% de absorciones de cada disolución, al que se le resta el fondo espectral de la radiación, con el eje de abscisas proporciona directamente la concentración de cromo en el cemento, expresada en $\mathrm{mg}$ de $\mathrm{Cr}$ por litro de disolución.

b) Determinación por el método de lectura directa

El contenido de compuestos de cromo en el cemento se puede conocer por determinación del \% de absorción de la muestra disuelta y preparada según se describe en el apartado "2,5. Técnica de trabajo", con el auxilio de la gráfica de calibrado. 
Gráfica de calibrado

La gráfica de calibrado, representada en la figura 5, se ha obtenido a partir de los resultados de una serie de nueve disoluciones de Cr (VI) preparadas a partir de la disolución patrón. Las nueve disoluciones tienen una concentración de $\mathrm{Cr}$ (VI) de 0,0, 0,1, 0,2, 0,3, 0,4, 0,5, 0,6, 0,8 y 1,0 mg por litro de disolución y $1 \%$ de La (III).

El volumen ha sido de $50 \mathrm{ml}$.

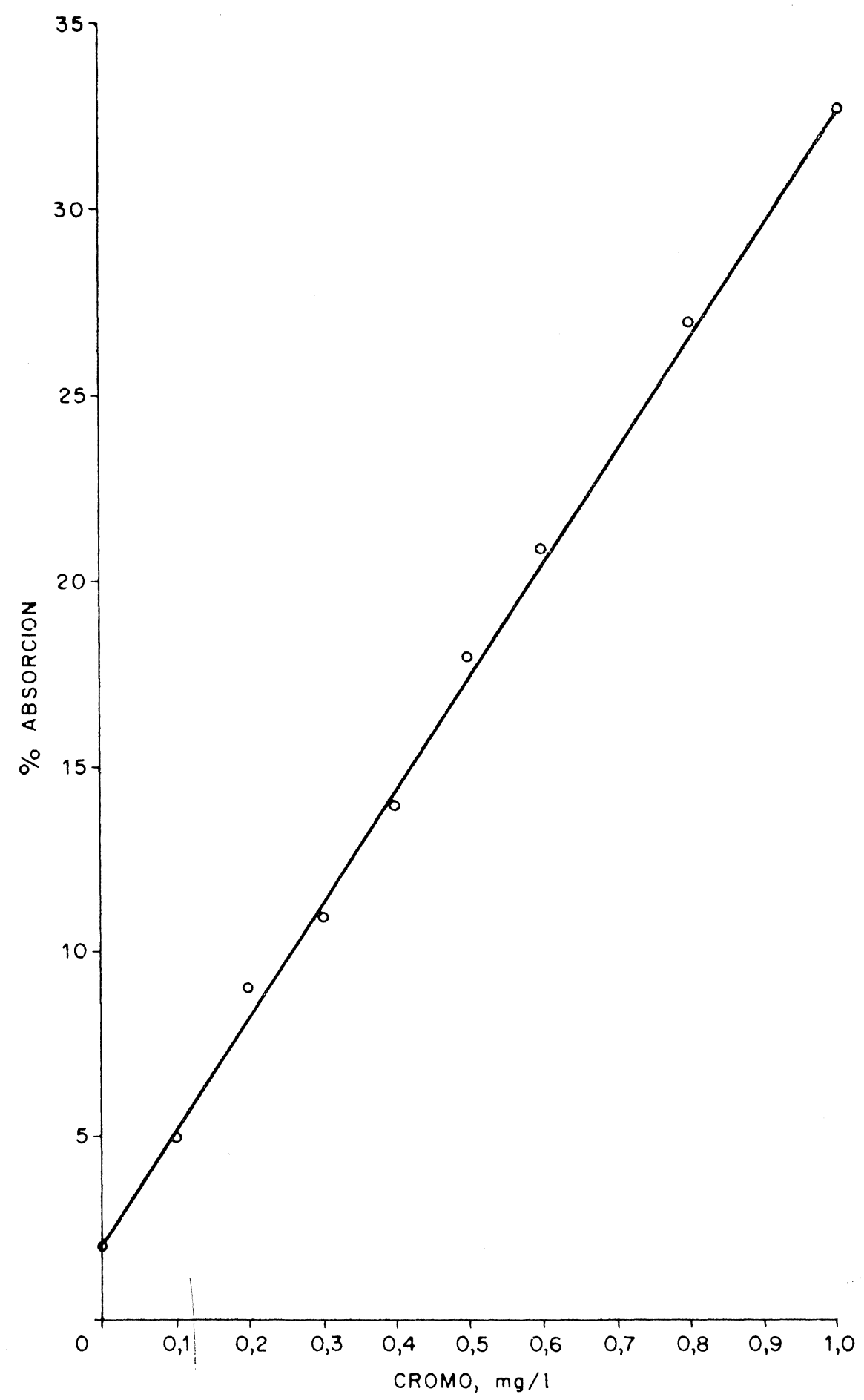

Fig. 5.-Cráfica de calibrado: \% absorción-cromo, $\mathrm{mg} / \mathrm{l}$. 


\section{Resultados}

Los resultados obtenidos, referidos a la muestra seca a $105^{\circ} 110^{\circ} \mathrm{C}$ y expresados en ppm, figuran en la tabla 3 .

El contenido de cromo por el método de lectura directa se ha determinado en seis muestras de las que venimos estudiando, seleccionadas por su contenido de cromo: 96,5, 55,5, $74,0,70,3,30,5$ y 55,0 ppm. En cada muestra se han realizado dos determinaciones distintas.

T A B L A 3

Contenido de cromo en 6 muestras de cemento

\begin{tabular}{cccc}
\hline & \multicolumn{3}{c}{ Cromo, ppm } \\
\cline { 2 - 4 } Muestra n.0 & \multicolumn{3}{c}{ Determinación } \\
\cline { 2 - 4 } & \multicolumn{2}{c}{ Valor medio } \\
\hline 1 & 94,0 & 93,5 & 93,8 \\
4 & 53,6 & 54,0 & 53,8 \\
5 & 72,5 & 72,0 & 72,3 \\
6 & 70,0 & 70,3 & 70,2 \\
9 & 27,8 & 28,5 & 28,2 \\
10 & 54,0 & 54,3 & 54,2 \\
\hline
\end{tabular}

\subsection{Estudio comparativo de los métodos de las adiciones patrón y de lectura directa}

En la tabla 4 se incluyen los resultados, ppm de cromo, de 6 muestras distintas de cemento, en donde puede apreciarse que los valores obtenidos por el método de las adiciones patrón son superiores a los obtenidos por el método de lectura directa en un valor, \% de ppm, comprendido entre 0,1 y 3,1 para las muestras cuyo contenido es superior a 50 ppm e inferior a $100 \mathrm{ppm}$. La diferencia mayor corresponde a la muestra con menor contenido de cromo, 28,2, 30,2 ppm; estos valores se encuentran en la zona inferior de la escala de trabajo, método de lectura directa, en donde, además, la absorción producida por los elemenos mayoritarios se hace más acentuada.

Como es natural, a pesar de la compensación de los efectos interelementales y de la matriz en el método de las adiciones patrón, la precisión absoluta de los valores va afectada de un determinado error al obtenerse por extrapolación.

\subsection{Sensibilidad}

La sensibilidad, concentración del ion en $\mu \mathrm{g} / \mathrm{ml}$ que produce una absorción del $1 \%$, es $0,003 \mu \mathrm{g} / \mathrm{ml} / 1 \%$ para las condiciones de trabajo señaladas en el apartado 2.1. 
T A B L A 4

Cromo, ppm, en 6 muestras de cemento. Estudio comparativo entre el método de las adiciones patrón y el de lectura directa

\begin{tabular}{ccccc}
\hline & \multicolumn{3}{c}{ Método } & \\
\cline { 2 - 3 } Muestra n.o & Lectura directa (1) & adiciones (2) & ppm (2)-(1) & \% referido a (1) \\
\hline 1 & 93,8 & 96,5 & 2,7 & 2,9 \\
4 & 53,8 & 55,5 & 1,7 & 3,1 \\
5 & 72,3 & 74,0 & 1,7 & 2,4 \\
6 & 70,2 & 70,3 & 0,1 & 0,1 \\
9 & 28,2 & 30,5 & 2,3 & 8,2 \\
10 & 54,2 & 55,0 & 0,8 & 1,5 \\
\hline
\end{tabular}

\subsection{Precisión}

Para conocer la precisión de la determinación del contenido de compuestos de cromo en el cemento por absorción atómica, método de lectura directa, se han realizado para una misma muestra, preparada en condiciones análogas, diez determinaciones. El estudio se ha hecho, por otra parte, en dos cementos y en 3 clínkeres de cemento portland.

La composición química de las muestras estudiadas se incluye en la tabla 1. La composición de la muestra n. ${ }^{\circ} 6$ figura en la tabla 1 de la publicación (1).

Los resultados obtenidos, cantidad de cromo en cada determinación expresada en ppm y referida a la muestra seca a $105^{\circ}-110^{\circ} \mathrm{C}$, así como el valor medio, $\bar{x}$, la desviación estándar estimada, $s$, la precisión, $\varepsilon$, el intervalo de confianza y el coeficiente de variación, C.V., figuran en la tabla 5 .

En dicha tabla puede apreciarse que la dispersión que existe en las medidas efectuadas en cada una de las diez determinaciones de una muestra es: de 4 ppm para la muestra de cemento n. 6 que tiene un contenido medio de $68,8 \mathrm{ppm}$, de $6 \mathrm{ppm}$ para la muestra de cemento $n .^{\circ} 11$ y las de clínker n. ${ }^{\circ 5} 12$ y 13 con un contenido de cromo de 48,1, 93,4 y $110,9 \mathrm{ppm}$, respectivamente, y de $12 \mathrm{ppm}$ para la muestra de clínker $\mathrm{n} .^{\circ} 14$ que tiene 112,1 ppm de cromo.

La desviación estándar estimada, $s$, es $1,93,2,26,2,46$ y 2,84 ppm para las muestras n. ${ }^{\text {s }} 6$, 12,13 y 11 , respectivamente, y 6,0 ppm para la muestra n. ${ }^{\circ} 14$, que presenta la mayor dispersión en las lecturas efectuadas.

El contenido de cromo se determinó por espectrofotocolorimetría en la muestra no 6 , de la que se realizaron 10 determinaciones distintas, fundiendo con $\mathrm{LiBO}_{2}$. La desviación estándar estimada, $s$, y el coeficiente de variación, C. V., 2,23 y 3,18\%, son mayoresr qúe cuando la determinación se hace por absorción atómica, 1,93 y $2,76 \%$.

\section{3.\%. Estudio comparativo de la determinación de compuestos de cromo por espectrofoto- colorimetría y por espectrofotometría de absorción atómica}

En la tabla 6 se incluyen los valores medios de los compuestos totales de cromo en las diez muestras de cemento portland estudiadas por espectrofotocolorimetría del complejo cromo (VI)-s. difenilcarbacida y por espectrofotometría de absorción atómica, método 
de las adiciones patrón. Los valores obtenidos por absorción atómica son superiores; incremento que se acentúa en aquellas muestras cuyas lecturas se encuentran en los extremos de las escalas de los equipos de trabajo utilizados, que corresponden a la zona no lineal de la gráfica de calibrado, de un modo especial en el método espectrofotocolorimétrico.

TABLA 5

Determinación de cromo en cementos por EAA. Precisión

\begin{tabular}{|c|c|c|c|c|c|}
\hline \multirow[b]{2}{*}{ Determinación } & \multicolumn{5}{|c|}{ Muestra $n \cdot{ }^{\circ}$} \\
\hline & 6 & 11 & 12 & 13 & 14 \\
\hline 1 & 70,0 & 46,0 & 93,0 & 113,0 & 107,0 \\
\hline 2 & 66,0 & 52,0 & 97,0 & 110,0 & 107,0 \\
\hline 3 & 70,0 & 49,0 & 91,0 & 113,0 & 107,0 \\
\hline 4 & 70,0 & 46,0 & 97,0 & 113,0 & 107,0 \\
\hline 5 & 70,0 & 46,0 & 93,0 & 113,0 & 107,0 \\
\hline 6 & 70,0 & 52,0 & 95,0 & 113,0 & 119,0 \\
\hline 7 & 66,0 & 46,0 & 91,0 & 110,0 & 110,0 \\
\hline 8 & 70,0 & 46,0 & 93,0 & 107,0 & 119,0 \\
\hline 9 & 66,0 & 46,0 & 91,0 & 110,0 & 119,0 \\
\hline 10 & 70,0 & 52,0 & 93,0 & 107,0 & 119,0 \\
\hline$\Sigma=$ & 688,0 & 481,0 & 934,0 & $1.109,0$ & $1.121,0$ \\
\hline Media, $\bar{x}=\frac{\Sigma x}{N}=$ & 68,8 & 48,1 & 93,4 & 110,9 & 112,1 \\
\hline $\begin{array}{l}\text { Desviación estándar estimac } \\
s=\sqrt{\frac{\sum(x-\bar{x})^{2}}{N-1}}=\end{array}$ & 1,93 & 2,84 & 2,26 & 2,46 & 6,0 \\
\hline Precisión, $\varepsilon=$ & 1,45 & 2,13 & 1,70 & 1,80 & 4,51 \\
\hline Intervalo de confianza $=$ & $68,8 \pm 1,45$ & $48,1 \pm 2,13$ & $93,4 \pm 1,70$ & $110,9 \pm 1,80$ & $112,1 \pm 4,51$ \\
\hline $\begin{array}{l}\text { Coeficiente de variación, } \\
\mathrm{CV}=\frac{s}{\bar{x}} \cdot 100\end{array}$ & $2,76 \%$ & $5,82 \%$ & $2,46 \%$ & $2,25 \%$ & $5,35 \%$ \\
\hline
\end{tabular}

Consideramos que la diferencia en los valores se debe, en gran parte, a una oxidación incompleta del $\mathrm{Cr}$ (III) y a los errores propios de la separación de los precipitados de hidróxidos.

Los valores obtenidos para un mismo cemento, hechos diez veces distintas por espectrofotocolorimetría, a partir de la muestra disgregada con $\mathrm{LiBO}_{2}$, tabla 5 de la primera parte (1), presentan una dispersión mayor, $61,5 \mathrm{ppm}$ a $74,5 \mathrm{ppm}$, que los obtenidos por espectrofotometría de absorción atómica por lectura directa, tabla 5, 66,0 ppm a 70,0 ppm; del mismo modo, el coeficiente de variación, C.V., es mayor $3,18 \%$ frente a $2,76 \%$, así como la desviación estándar estimada, $s, 2,23$ frente a 1,93 . 
TAB L A 6

Contenido de compuestos de cromo en 10 muestras de cemento. Determinación por espectrofotocolorimetría y por espectrofotometría de absorción atómica

\begin{tabular}{ccccc}
\hline & \multicolumn{2}{c}{ Cromo, ppm } & & \\
\cline { 2 - 4 } Muestra n.o & $\begin{array}{c}\text { Espectrofoto- } \\
\text { colorimetría (1) }\end{array}$ & $\begin{array}{c}\text { Absorción atómica } \\
\text { M. adiciones patrón (2) }\end{array}$ & ppm (2)-(1) & \% referido a (2) \\
\hline 1 & 91,5 & 96,5 & 5,0 & 5,1 \\
2 & 50,5 & 53,0 & 2,5 & 4,7 \\
3 & 32,3 & 35,3 & 3,0 & 8,5 \\
4 & 56,1 & 55,5 & $-0,6$ & 1,1 \\
6 & 71,3 & 74,0 & 2,7 & 3,6 \\
7 & 69,6 & 70,3 & 0,7 & 1,0 \\
8 & 75,4 & 75,0 & $-0,4$ & 0,5 \\
9 & 47,6 & 48,0 & 0,4 & 0,8 \\
10 & 24,0 & 30,5 & 6,5 & 21,3 \\
\hline
\end{tabular}

A la vista de los resultados obtenidos consideramos que la espectrofotometría de absorción atómica se puede utilizar para determinar el contenido total de compuestos de cromo, $\mathrm{Cr}$ (III) y $\mathrm{Cr}$ (VI), en el cemento portland, tomando las debidas precauciones - cantidad de muestra - para que dicho contenido se encuentre en la zona de errores menores.

En resumen, la preparación de la muestra para el método de absorción atómica, tanto para el de adiciones patrón como de lectura directa, es más rápida, precisa menos operaciones y no presenta las dificultades correspondientes a la oxidación cuantitativa del $\mathrm{Cr}$ (III) a $\mathrm{Cr}$ (VI) y a la separación del Fe (III) que necesita el método espectrofotocolorimétrico.

Por otra parte, la muestra preparada para utilizar la técnica de absorción atómica se puede emplear, con las precauciones debidas, para determinar los iones $\mathrm{Ca}$ (II), $\mathrm{Mg}$ (II), $\mathrm{Al}$ (III), Fel (III), Si (IV), Mn (II), Sr (II), Ti (IV), Na (I) y K (I) del cemento portland.

\section{CONCLUSIONES}

Primera. El contenido total de compuestos ide cromo, Cr (III) y Cr (VI), en el cemento portland se puede determinar por espectrofotometría de absorción atómica, bien por el método de adiciones-patrón o bien por el de lectura directa, disgregando la muestra con $\mathrm{LiBO}_{2}$.

Segunda. Los valores obtenidos por espectrofotometría de absorción atómica son ligeramente superiores a los que se obtienen por espectrofotocolorimetría del complejo coloreado formado por reacción del $\mathrm{Cr}$ (VI) con la s-difenilcarbacida en medio $\mathrm{H}_{2} \mathrm{SO}_{4}-0,2 \mathrm{~N}$; sin embargo, la dispersión, la desviación estándar estimada y el coeficiente de variación son menores. 
Tercera. La determinación de compuestos de cromo en el cemento portland por espectrofotometría de absorción atómica es una técnica de trabajo más simple y más rápida que la espectrofotocolorimétrica; por otra parte, se puede incluir como una operación más en el análisis del cemento por la mencionada técnica de absorción atómica, siendo comunes la disgregación, con $\mathrm{LiBO}_{2}$, y la eliminación de interferencias con La (III).

\section{B I B L I O G R A F I A}

(1) Gaspar-Tebar, D.; Triviño-Vazquez, F. y Alvarez-Puente, V.: Materiales de Construcción 163, 27-41, (1976).

(2) Barnes, L.: Anal. Chem., 8, 1.085 (1966).

(3) Giammarise, A.: Atomic. Absorpt. Newsletter, 5, 113, (1966).

(4) Takeuchi, T. and Suzuki, M.: Talanta, 11, 1.066; según Tenoutasse, loc. cit.

(5) Crow, R. F., Hime, W. G. and Connolly, J. D.: J. of the PCA Res. and Dev. Lab. vol. 9, 2, 60, (1967).

(6) Roos, J. T. H. and PrICE, W. J.: Analyst, 94, 89, (1969); segn Bonomi y otros, loc. cit.

(7) Tenoutasse, N.: Rev. des Mat. de Const., 664-665, 666, 668, 1-9, 65-72 y 139-144, (1971).

(8) Bonomi, F.; Francardi, M. T. e Massazza, F.: Il Cemento, 3, 163-178, (1972).

(9) Skoog, D. A. y WeSt, D. M.: Análisis Instrumental. Edit. Interamericana 1. $^{a}$ edición en español (1975), pág. 130.

(10) Fifiedd, F. W. and Kealy, D.: Analytical Chemistry. Published by Intern. Textbook Co Ltd. London (1975), pág. 234.

(11) Boar, P. L. and Ingram, L. K.: Analyst, vol. 95, 124-130, (fev. 1970), según Tenoutasse, loc. cit.

(12) Medinn, J. H.; Suhr, N. H. and Budkin, J. B.: Atomic Absorption Newsletter, 2, 25-29, (1969); según BoNOMI y otros, loc. cit.

(13) VAN Loon, J. C. and Parissis, C. M.: The Analyst, 94, 1.057-1.062, (1969), según Bonomi y otros, loc. cit.

(14) Crow, R. F. and Connolly, J. D.: J. of Test. and Eral. ITEVA, vol. 1, 5, 382-383, (1973).

(15) Govindarajin, K.: Analysis, 2, 367-376, (1973).

(16) Degre, P.: Rev. des Mat. 685, 33-36, (1973).

(17) Hameau, Mb.: Afinidad, 237, 177, (1965).

(18) Triviño, F.: Atomic Absorpt. Newsletter, vol. 14, 3, 70, (1975).

(19) PCCH-64: IETCcc. Madrid (1964), págs. 39-45.

(20) Valle, J.: Tesis Doctoral A-60; Fac. Ciencias Univ. Complutense. Madrid, (marzo, 1966).

(21) KEIL, F.: Cemento. Edit. Técnicas Asociadas, S. A. Barcelona (1973), págs. 72-73.

(22) Tenoutasse, N.: Rev. des Mat. de Const. 666, 67, (1971).

(23) Bonomi, F.; Francardi, M. T. e Massazza, F.: loc. cit., pág. 71. 